\title{
DESIGN AND IMPLEMENTATION OF DIGITAL AUDIO BROADCASTING TRANSMITTER AND RECEIVER USING RAYLEIGH FADING CHANNEL
}

\author{
Drakshayini M. $\mathbf{N}^{1}$, Aishwarya $\mathrm{A}^{2}$, Archanag ${ }^{3}$,Divya $\mathbf{H}^{4}$,Fouzia Tabassum ${ }^{5}$ \\ ${ }^{1}$ Associate Professor, Department of Electronics and Communication, TJIT, Bangalore, Karnataka, India. \\ ${ }^{2}$ Students, (B.TECH) of TJIT, Bangalore, Karnataka, India.
}

\begin{abstract}
In this paper, we proposed to implement the Digital Audio Broadcasting-(DAB) Transmitter and receiver part. The main problem in frequency modulation is multipath fading and ISI. To solve this problem we proposed the use of DAB with OFDM system. A matlab-simulink model is designed for DAB. The well defined radio equipment designed to broadcast the radio stations provide high-class data and audio forces to transportable receivers. The DAB conduction mode-II is executed first and then it is extended to other modes successfully. We take any audio as input and calculate the BER between transmitted and received signal. As a part of this study, execution criteria for Rayleigh fading channel has been led for BER.
\end{abstract}

Keywords: orthogonal frequency division multiplexing, bit error rate, Quadrature phase shift multiplexing, Digital Audio Broadcasting

\section{INTRODUCTION}

Digital_Audio Broadcasting (DAB) has made by the European consortium Eureka 147 in mid 1990's, basically substitute the general used straightforward simple recurrence balance (Frequency Modulation) Television system. The Very High Frequency (VHF) band is an uncommon source in various part of the globe, therefore requirement for promptly accessible necessity for an terribly more powerful regulation procedure than "FM".DAB is another computerized radio configuration that passes on radio projects from the studio to the recipient. DAB is proposed to pass on top feature advanced sound undertakings and data organizations to altered, versatile and convenient beneficiaries which can utilize clear whip radio wires. It was created in the 1990's by the Eureka_147 Digital_Audio Broadcasting venture. Digital_Audio Broadcasting is a great degree fitting for convenient gathering and gives high toughness against multiple path gathering. It grants usage of Single Frequency Networks (SFNs) for high recurrence viability. Digital Audio Broadcasting uses COFDM innovation that makes it impenetrable to multiple path blurring and Intersymbol Interference (ISI). FM gathering can be extremely impacted by shadowing and by dormant echoes (the arrival in the collector of put off "multiple path" signals which receiver of postponed "multiple path" signals which are reflected from elevated buildings, mountains and slopes).

\section{DAB TRANSMISSION SYSTEM}

The general Digital Audio Broadcasting transmission framework can be isolated into different sub-hinders as appeared in figure 1. The sound indicator is Moving Picture Expert Group layer-2 encoded and a short time later blended utilizing scrambler. Forward mistake rectification is joined to the scrambler bit-stream by using punctured convolutional codes with code-rates. The bit-stream is sent through a period Interleaver sooner than bits are multiplexed with substitute projects to shape a gathering. The assembled bit stream is separated into individual Orthogonal Frequency Division Multiplexing images, which are accomplished by differential BPSK modulation of the subcarriers and basically a converse Fourier change (IFFT) function within the Orthogonal Frequency Division Multiplexing transmitter. In the collector the relating reverse operations must be finished. The bit stream of the information is parcelled into bit streams of lower rate in Orthogonal Frequency Division Multiplexing, which are independently adjusted onto orthogonal sub-transporters. To fulfil orthogonality sub-bearers are partitioned in recurrence by the backwards of the image length, hypothetically achieving zero intersymbol impedance (ISI).

In spite of the fact that the sinc(f) reactions commonly have common characteristics, they experience zero at centre frequencies of all other sub-carriers, giving band effectiveness for BPSK modulation of every sub-carrier. Orthogonal sub-carriers can be approved using IFFT algorithm, which can be eagerly included in hardware. Every sub-carrier is modulated with BPSK, which maps the arriving bits to intricate symbols for every sub-carrier K. 


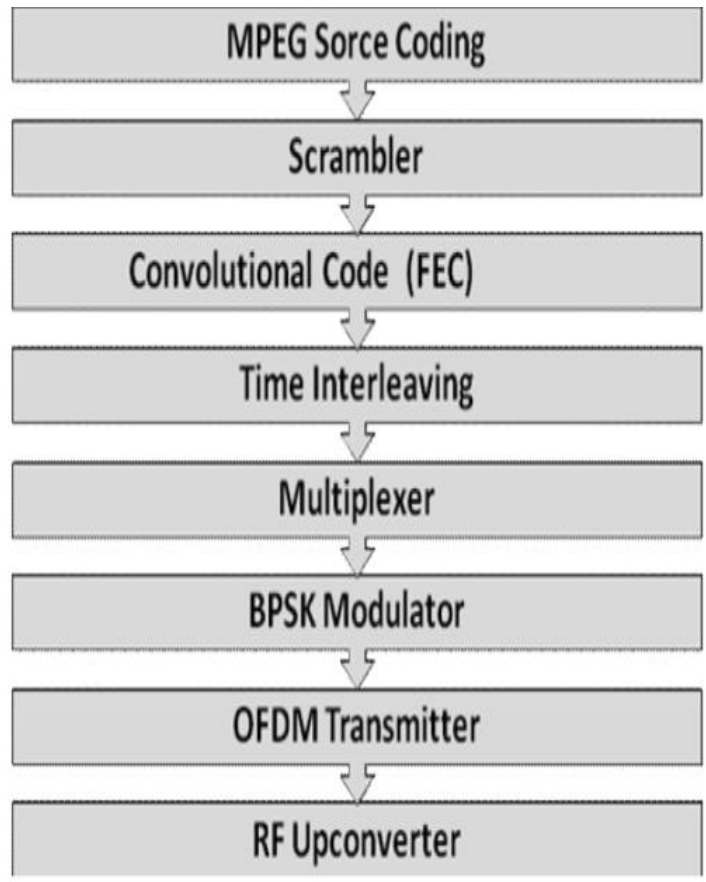

Figure 1: DAB functional diagram of transmitter

\section{DAB TRANSMISSION MODES:}

Actually, the DAB transmission framework know how to be utilized as a part of all VHF and UHF television recurrence groups between 30 and $3000 \mathrm{MHz}$ and four particular modes for run of the great applications have been characterized (Table I). The aggregate image length comprises of the foremost image period also, a gatekeeper interim, which keeps the reverberation of the past image from interfering with the current image. Thusly, between image impedance (ISI) is lessened to right around zero the length of the echoes from the different transmitters and engendering ways don't significantly surpass the gatekeeper interim.

\section{TABLE 1}

THE DAB TRANSMISSION MODES

\begin{tabular}{|l|c|c|}
\hline Mode & $\begin{array}{c}\text { Total } \\
\text { symbol } \\
\text { duration }\end{array}$ & Main application \\
\hline I. & 1246 us & $\begin{array}{c}\text { Terrestrial DAB,Large } \\
\text { coverage area,VHF. }\end{array}$ \\
\hline II. & 312 us & $\begin{array}{c}\text { Terrestrial DAB,small } \\
\text { to medium coverage } \\
\text { area,UHF. }\end{array}$ \\
\hline III. & 156 us & $\begin{array}{c}\text { Satellite DAB,no long } \\
\text { echoes,UHF. }\end{array}$ \\
\hline IV. & 623 us & $\begin{array}{c}\text { For Canada, between } \\
\text { mode 1 and 2. }\end{array}$ \\
\hline
\end{tabular}

The nearer the sub-carriers are divided the more serious gets be be intercarrier abstraction,thus image term is a trade of .that is if image span is too short then differ spread of the channel causes intersymbol impedance while if the image length is too long then sub-carries turn out to be firmly separated in recurrence empowering officially small Doppler movements to deliver high intercarrier obstruction. Inside of the gathering data transmission contrasts from one mode to another. In the event that the beneficiary physically moves inside of the gathering region, Doppler spread increments and worldly soundness of the channel is decreased. What's more the signal range is Doppler moved. On the off chance that they got OFDM sub-transporters are moved concerning the reference recurrence in the beneficiary, between bearer obstruction is expanded.

The nearer the sub-transporters are separated, the more serious gets to be between transporter obstruction, thusly image term is a trade off: i.e. on the off chance that image term is very smaller than the suspended spread of the channel causes intersymbol obstruction, while if image span is too long then sub-bearers turn out to be nearly separated in recurrence empowering effectively little Doppler movements to deliver far above the ground intercarrier impedance. While if image term is moreover extended then sub-transporters get to be firmly separated in recurrence empowering effectively little Doppler movements to deliver high intercarrier impedance.

\section{SIMULINK_MODEL IN MATLAB INFORMATION_SOURCE}

Figure 2 shows the block diagram for transmitter and receiver.This is the main square in the transmitter area of the Touch framework model for reproduction. It produces arbitrary paired information bit succession for FIC and MSC. Consequently the equation 1 gives the information for one transmission edge that is given by:

DATA bits=FIC(DATA $)+$ MSC (DATA $)$

MSC is a period interleaved information path isolated within a number of sub path which are independently convolutional coded. FIC is utilized to flag the complex setup of the DAB communication and administration data.

\section{CONVOLUTIONAL_ENCODER}

Convolutional_encoder encodes a series of double information vectors to create an arrangement of parallel yield vectors. This piece can handle various images at once. Here the yield information stream transmitted bits from past piece is information to convolution encoder. Path coding depends on punctured convolutional self-assured mistake rectification (FEC) which permits both unequal and equal Error Protection (UEP) portrayed in segment. Touch framework has a convolutional_encoder with requirement length 7 and 133,171, 145 and 133 are the octal types of generator polynomials separately. 


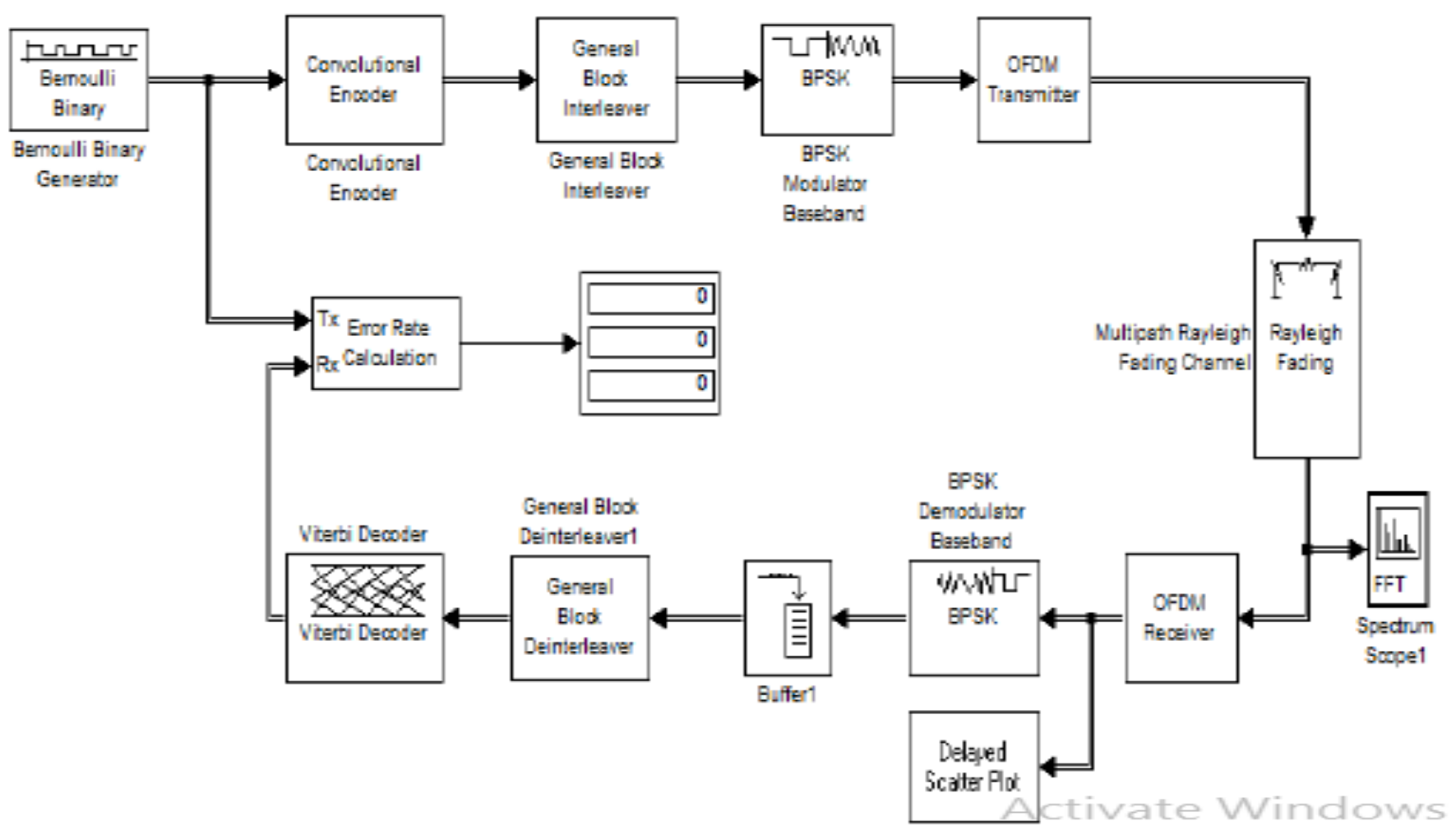

Figure2: Simulink model of DAB transmitter and receiver

\subsection{General-Block Interleaver}

The General-Block Interleaver piece improves the components of its information vector not including rehashing or discarding any components. On the off chance that the information contains $\mathrm{N}$ components, at that moment the Elements factor is a section vector of duration $\mathrm{N}$.

\subsection{Bpsk_Modulator}

Portable correspondences the multiple path impact will debase the period of bearers. The answer for that issue is to convey the data as the distinction sandwiched between the periods of two images. This is proficient by the part Binary Phase Shift Keying balance. In this tweak plan there is no supreme stage indication, every image is referenced just against the past image. This extraordinarily improves the decoder.

\subsection{Qpsk_Mapping}

Quadrature Phase Shift Keying computerized image mapping square is dependable for mapping (in parallel) serial flow in every information hinder within computerized group of stars as indicated by QPSK adjustment plan shown within Digital Audio Broadcasting standard characterized as every information square of 768 bits is mapped onto the 384 complex coefficients for one Orthogonal Frequency Division Multiplexing image of Time Span. The initial 384 bits will be mapped to the genuine parts of the 384 QPSK images; the last 384 bits will be mapped to the nonexistent parts. QPSK encodes two bits for each image. So the each block contains the 75 blocks out of 384 parts which is its composite yield.

\subsection{Frequency De-Interleaving}

Recurrence interleaving action is done at the transmitter to invalidate transmission aggravations, for example, specific distort. This secondary-part performs the converse of the recurrence interleaving by re-course of action of the bits to get Quadrature Phase Shift Keying image piece indistinguishable with yield of Quadrature Phase Shift Keying mapping.

\subsection{QPSK De-Mapping}

Secondary-piece change over to get complex Quadrature Phase Shift Keying images from the recurrence deInterleaver yield into bits. At point when indication of the component of the multifarious Quadrature Phase Shift Keying image is negative, the decoded bit is 1 and when it is constructive decoded bit is 0 . Identical guideline is relevant to nonexistent part of the complex QPSK image. The decoded bits has to be orchestrate in the comparable way in which bit was utilized for Quadrature Phase shift Keying mapping. The decoded I stage part data will be allotted to record 1 to 384 and Q stage segment bits to record 385 to 768.

\subsection{Viterbi_Decoder}

Viterbi_Decoder square disentangles the information images to produce dual yield images. This square can handle a few images at once for the faster execution. The information to Viterbi_decoder will be tough-chosen bits those are 0 or 1 . 


\section{RESULT}

Figure 4 presents the result of spectrum scope. It is mixture of signal from Rayleigh Fading and OFDM.

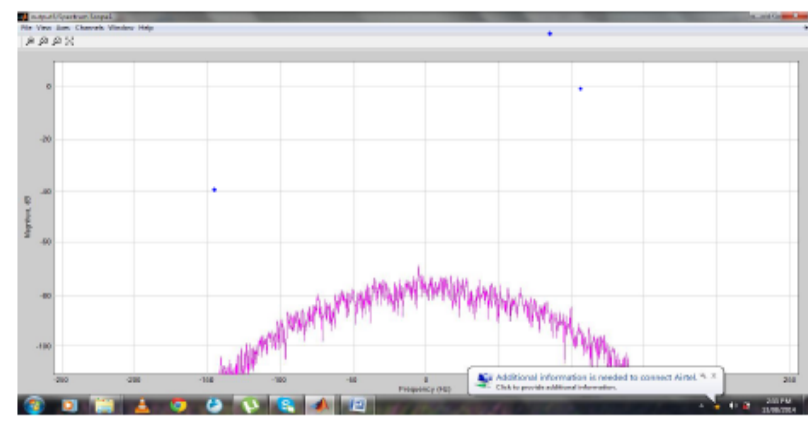

Fig 3. Output of spectrum scope

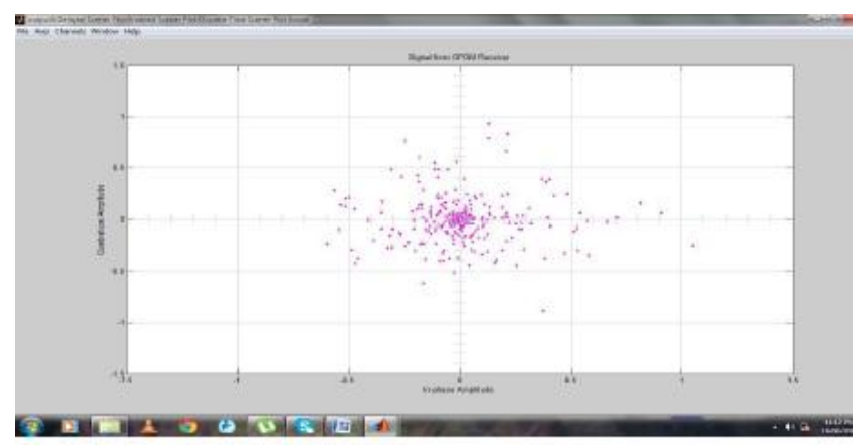

Fig 4. Constellation when Doppler shift is 5

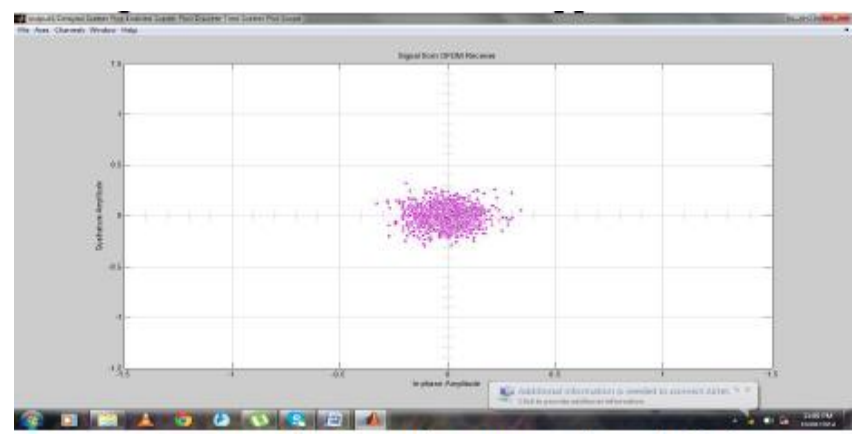

Fig 5. Constellation when Doppler Shift is 50

\section{CONCLUSION}

The execution of DAB adjustment plans Outline based should be utilized to display multi-rate frameworks for example, Digital Audio Broadcasting. Hereafter the Digital Audio Broadcasting framework will have the different degree while the audience greet another innovation which offers more decision and higher specialized quality, and in addition an extremely powerful flag while hearing the programmes in a means handling of expression or on a compact position. Another recurrence distribution arrangement at the VHF have concurred the Europe. Digital Audio Broadcasting gives adequate frequencies to begin the physical Digital Audio Broadcasting administrations. And be anticipated under particular manufactured analysis circumstances with the Matlab-Simulink. The graphical client interface empowers the client to alter parameters quickly and to get a quantitative feel with respect to how transmission quality is influenced if these parameters are balanced. Finding the connection between bit mistake proportion and subjective sound quality at an early stage prompts effective listening tests are maintained a strategic distance execution estimation since broad from. Reproduction in the composite baseband space is wellmatched to foresee the execution of balance plans counting various types of channels.

\section{REFERENCES}

[1]. ETSI EN 300401, "Radio Broadcasting Systems; Digital Audio Broadcasting (DAB) to mobile,portable and fixed receivers "European Telecommunications Standards Institute, France, 2001.

[2]. S. Fechtel, "A Novel Approach to Modeling and Efficient Simulation of Frequency-Selective Fading Radio hannels", IEEE Journal on Selected Areas in Communications, Vol. 11, No. 3, New York, April 1993.

[3]. B. Le Floch, R. Halbert-Lassalle, D. Castelain, "Digital Sound Broadcasting to Mobile Receivers", IEEE Transactions on Comsumer Electronics, Vol. 35, No. 3, New York, August 1989. Glover, P. M. Grant, "Digital Communications", Prentice Hall Europe, England, p. 470, 1998.

[4]. J. G. Proakis, "Communication Systems Engineering", Prentice Hall International Inc., London, pp. 699-702, 1994.

[5]. Martoyo, H.Sobher, F.Jondral, CDMA vs OFDM; A performance comparison in selective fading channels, IEEE 7th International Symposium on spread spectrum technology and application, Prague, Czech Republic, page 139, Sept. 2002

[6]. Satish Singh \& Rakesh Ranjan Pani, Software Implementation Ofothogonal Frequency Division Multiplexing (Ofdm) Scheme For Mobile Radio Channel

[7]. Vishwas Sudaramurthy, A software simulation tested for CDMA wireless communication systems, Rice University, Houston, Texas, May 1999.

[8]. Mrutyunjay Panda, Dr. Sarat K.Patra, Simulation study of OFDM, COFDM, MIMO-OFDM Systems, Sensors and Transducer Journal, Vol 106, Issue 7, July 2009.

[9]. Sravankumar Veerapu,Vivek Chandran,Jayaram Navaneeth Malineni Performance evaluation of higher order modulations in OFDM systems,2013.

[10]. Yong Soo Cho, Jaekwon Kim, Won Young Yang, and Chung-Gu Kang. MIMO-OFDM Wireless Communications With MATLAB. Wiley, 2010.

[11].Hiroshi Harada and Ramjee Prasad. Simulation and Software Radio for Mobile Communications. Artech House on Demand, 2002. 


\section{BIOGRAPHIES}

1. Dr. Arun Vikas Singh received M.Tech \& Ph.D. in Electronics and communication Engineering. $\mathrm{He}$ is presently working as professor and HOD in Department of ECE at T.John Institute Of Technology.

2. Mrs. Drakshayini M. N, M.Tech in Department of ECE She is currently working as Associate Professor in TJIT

3. Students Archana G, Fouzia Tabassum, Aishwarya A, Divya $\mathbf{H}$ pursuing B Tech $\left(8^{\text {th }}\right.$ sem) ECE at TJIT 